\title{
Design of the UAV Digital Actuator Automatic Test System Based on Dual-core Microcontroller TMS320F28377D
}

\author{
Tao Yang ${ }^{*}$, Liren Chai and Gang Wang \\ China Academy of Aerospace Aerodynamics, Beijing, 100074, China \\ ${ }^{*}$ Corresponding author
}

\begin{abstract}
For purpose of UAV digital actuator test, one of UAV's key components, whose static and dynamic performance impacts on the UAV maneuvering performance, this paper studies an automatic digital actuator test system, introduces its hardware and software design scheme, and demonstrates its application. The TI dual-core microcontroller TMS28377D serves as its core with abundant communication interfaces widely used in digital actuators such as RS422, RS485, RS232, and CAN Bus. Besides, there are rotary incremental encoders connected to the test system, providing the actual position information of digital actuator, as well as a SD card for data record in real time. Via Ethernet communication, the test system communicates with the upper computer which sends command and receives feedback. The advantages of automatic digital actuator designed in this paper are high reliability and easy upgrading. The automatic test system has great potential in the development, production and storage of the UAV digital actuator.
\end{abstract}

Keywords-UAV; digital actuator; automatic test system; TMS320F28377D

\section{INTRODUCTION}

UAV, short for Unmanned Aerial Vehicle, is a reusable and controllable unmanned aircraft, carrying different payloads and executing different tasks. Thanks to its low cost, great reusability and high maneuver, UAV is widely used in military field, civilian area and scientific research [1]. As one of its key components, the actuator receives command from UAV flight control computer. The actuator is a typical servo system and it works as the control computer tells it to [2]. Its performance impacts on the UAV maneuvering performance severely. If not working properly, the UAV turns uncontrollable and stands a big possibility of crash on the ground, leading to heavy economic loss or even innocent casualty [3, 4]. As a prevailing trend, the digital actuator is now replacing the old-fashioned analog actuator. And this is what our research group is doing now. Although there are analog and PWM signal as the command and feedback signal for the current actuator, the newly developed actuator adopts digital signal for higher precision and better anti-interference ability. As a result, the corresponding automatic test system has to be designed. The automatic test system, which is pretty important in digital actuator debug, error detection and status indication during the whole process of development, production, storage and maintenance, provides the test support for the digital actuator and offers footstone of actuator reliable performance [5, 6]. As long as the digital actuator can pass all the tests, it can be mounted in the UAV for flight mission. What's more, with the advance of the UAV technology, high stability, high precision, fast response and long life are the essential qualities of the digital actuator. The test system had better be updated easily just by software modification without any change of the hardware.

\section{Digital Actuator Automatic Test System}

The controller and the electric actuator comprise the digital actuator. The controller is made up of different circuit with different functions, namely, communication interface, motor drive, power supply and digital control, while the electric actuator includes DC motor, drive reducer, output mechanism and potentiometer. As the core of the digital actuator, the controller communicates with the automatic test system via various digital communication interfaces. In the meanwhile, the automatic test system is linked to the upper computer for command transmission and data interaction. As shown in Figure I, the block scheme of system connection is presented. The digital interfaces between the digital actuator and automatic test system are RS422, RS485, RS232, and CAN Bus. There is the rotary incremental encoder mounted on the same axis of actuator output mechanism, and the reference of actual actuator position is given and sent back to the test system for the convenience of data analysis. Fast Ethernet communication circuit is the bridge of the automatic test system and the upper computer. The exclusive software developed in the upper computer sends command, receives data, and draws curves.

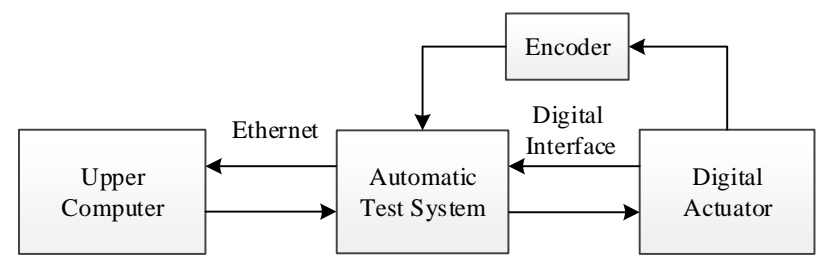

FIGURE I. SYSTEM CONNECTING BLOCK SCHEME

\section{THE HARDWARE DESIGN}

As for the hardware design, it is introduced in the form of modular design. The digital control core is the TI microcontroller TMS320F28377D with dual-core architecture. 
Power circuit offers power supply for different circuits in the system. The RS422/RS485/RS232 and CAN communication circuit conduct communication between the test system and the digital actuator. The Ethernet communication circuit connects the test system and the upper computer. The encoder circuit obtains position, direction and speed information from the rotary incremental. And the data storage circuit records the data online in real time via a SD card.

\section{A. Digital Control Core}

TMS320F28377D is a fast-speed and high-precision control chip with great reliability, in which two 32-Bit CPUs can cooperate together to provide $200 \mathrm{MHz}$ of signal processing performance in each one. It supports up to $1 \mathrm{MB}$ of onboard flash memory with error correction code and up to 204KB of SRAM. The circuit design is simplified by its rich communication and control peripherals, among which the SCI, eCAN, SPI, and eQEP modules are used. As shown in Figure 2, the SPI modules are connected to the Ethernet circuit and the SD card; the SCI modules are connected to RS422, RS485 and RS232 transceivers; the eCAN module is connected to the CAN transceiver; the eQEP module is connected to the rotary incremental encoder.

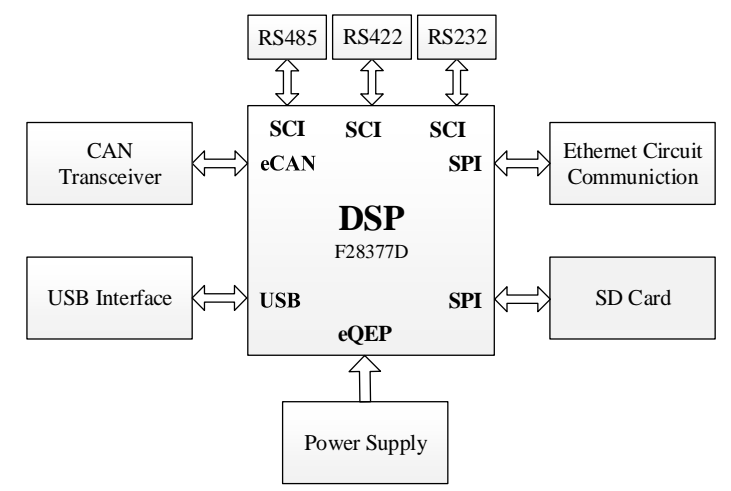

FIGURE II. BLOCK DIAGRAM OF THE DIGITAL CONTROL CORE

\section{B. Power Circuit}

The EMI filter helps eliminate the electromagnetic interference of DC power and then cleaner power supply $+5 \mathrm{~V}$ is obtained. As shown in Figure III, $+5 \mathrm{~V}$ is used to power up the CAN transceiver and the rotary encoder; the step down DC/DC converter TPS54331 is selected to transform $+5 \mathrm{~V}$ into $+3.3 \mathrm{~V}$ and $+1.2 \mathrm{~V}$ respectively to power up the control chip TMS320F28377D. And also +3.3V acts as the power source of the Ethernet controller and the RS422/RS485/RS232 transceiver.

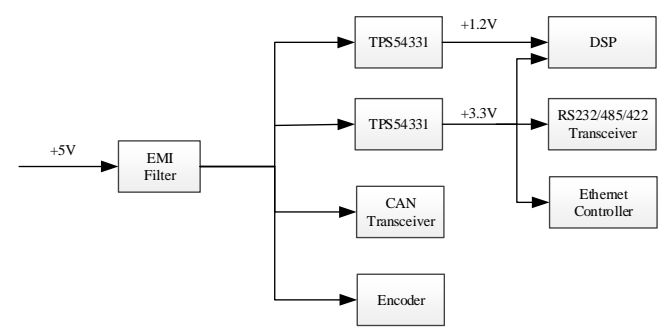

FIGURE III. SYSTEM POWER SUPPLY

\section{RS422/RS485/RS232 Communication Circuit}

Serial communication is used for all long-haul communication and it is becoming more and more common even at shorter distances because of improved signal integrity and transmission speed. The RS422, RS485 and RS422 are the typical standards for serial communication, which can be used in the digital actuator communication. So for high extensibility, the test system designs all these communication interfaces. As shown in Figure IV, MAX3490 serves as the RS422 transceiver in the communication circuit; MAX3485 serves as the RS485 transceiver in the communication circuit; MAX3232 serves as the RS232 transceiver in the communication circuit. For the simple reason that the three transceivers are able to operate form a single $+3.3 \mathrm{~V}$ supply, the SCITXD pin and SCIRXD pin of the F28377D SCI modules can directly connect to the transceivers' driver input and receiver output respectively.

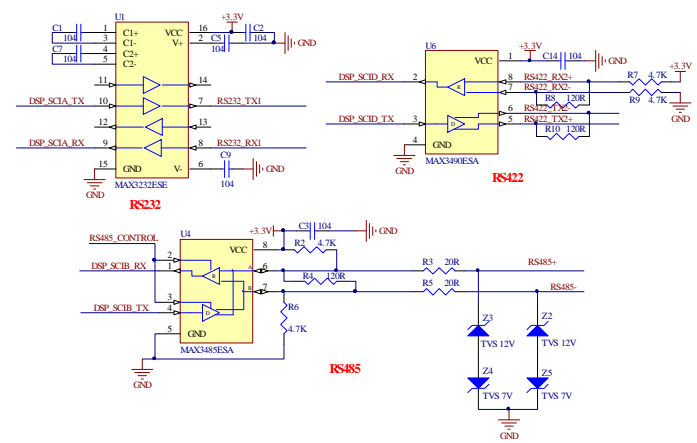

FIGURE IV. RS422/RS485/RS232 COMMUNICATION CIRCUIT

\section{CAN Communication Circuit}

The CAN bus is a robust message-based protocol, designed originally for multiplex electrical wiring within automobiles to save on cooper, but recently it is also used in many other contexts. The digital actuator is one of its application areas. As for TMS320F28377D, the CAN module is a serial communications protocol which efficiently supports distributed real-time control with a high level of reliability. The CAN module supports bit-rates up to $1 \mathrm{Mbit} / \mathrm{s}$ and is compliant with the ISO11898-1 (CAN 2.0B) protocol specification. In Figure V, it can be seen that fast CTM1050T with isolation is selected as the CAN transceiver. Despite the fact that it is powered by $+5 \mathrm{~V}$, the electrical level of its driver and receiver are compatible with $+3.3 \mathrm{~V}$, namely, the I/O level of TMS320F28377D. The CTM1050T can be connected to the CAN module directly via CAN_TX and CAN_RX.

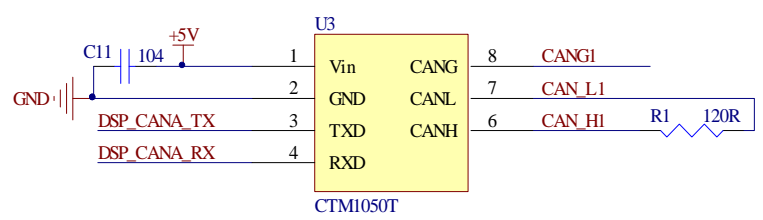

FIGURE V. CAN COMMUNICATION CIRCUIT 


\section{E. Ethernet Communication Circuit}

The communication between the test system and the upper computer has to be fast and stable. In this case, the Ethernet communication is a perfect choice and W5500 is selected as the Ethernet controller. The W5500 chip is a hardwired TCP/IP embedded Ethernet controller that provides easier Internet connection to the digital control core by SPI peripheral. W5500's SPI supports $80 \mathrm{MHz}$ speed and new efficient SPI protocol for the high speed network communication. W5500 embeds the 32Kbyte internal memory buffer for the Ethernet packet processing. It is fast and easy to implement the Ethernet application just by adding the simple socket program. As shown in Figure VI, the W5500's SPI works in the slave mode, and connects to TMS320F28377D SPI module via SCS, SCLK, MOSI and MISO. The TXN/TXP and RXN/RXP differential signals are linked to the HR91105A, a RJ45 connector with integrated magnetics.

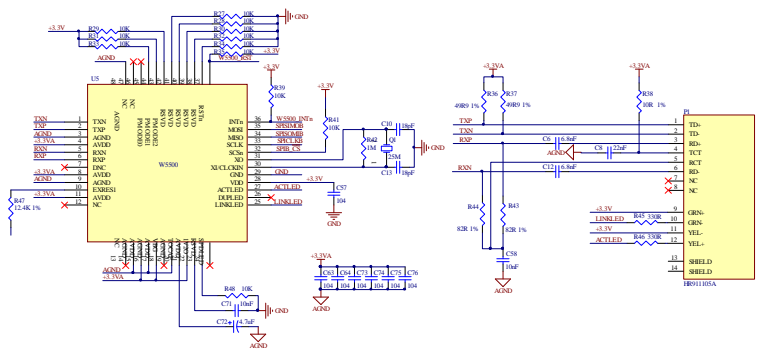

FIGURE VI. ETHERNET COMMUNICATION CIRCUIT

\section{The SOFTwARE DESIGN}

The software design is made up of two parts, the DSP embedded software and the upper computer software. The embedded software refers to software of the digital control core TMS320F28377D, while the upper computer software offers the human-machine interaction. The embedded software conducts digital communication control, whose flow is as follows. At first, after power up, the control core is reset. And then it initiates the whole system control, SCI module, eCAN module, SPI module, eQEP module and the Cputimer0. The relevant vector table of peripheral interrupt controller and the interrupt function is initiated. Enable the global interrupt and then enter the while (1) infinite loop. The transmission of different communication peripherals with different intervals are triggered in Cputimer0 interrupt. The digital data acquisition is conducted by the communication peripheral receiver interrupts. In this way, the reliability and real-time performance of the digital data acquisition is ensured. Different digital actuators have different communication protocol. In order to meet the requirement of universality and easy upgrading, the communication protocol analysis is conducted in while (1) function. The test system sends data to the upper computer via the Ethernet controller with a period of $1 \mathrm{~ms}$ and data length 40 bytes. As for the upper computer software shown in Figure VII, it transmits all kinds of commands to the automatic test system. After analyzing the commands, the test system sends corresponding commands to the digital actuator to perform automatic test. Also, it can accomplish data storage and draw curves of the data received in real time.

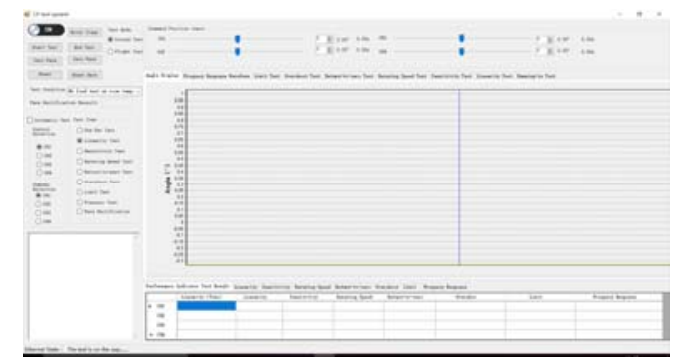

FIGURE VII. THE UPPER COMPUTER SOFTWARE

\section{CONCLUSION}

As UAV's key component, the digital actuator is a determinant factor for UAV performance. To find out whether the digital actuator works well or not before flight, the automatic test system is needed. In this paper, the automatic digital actuator test system is developed with the dual-core TMS28377D with high reliability as its control core. The automatic system facilitates digital actuator debug, error detection and status indication with rich communication interfaces in the whole life span of the digital actuator from development and production to storage and maintenance. Taking different factors into consideration when the automatic test system is designed, its advantages such as fast response, high reliability and easy upgrading are not hard to figure out, which is also verified by a recent digital actuator test with CAN communication interface. As more and more digital actuators designed in our research group, the application prospect of the automatic test system is apparently promising. Time will tell and prove its superiority in the digital actuator test field.

\section{References}

[1] Di Bin, Zhou Rui, Ding Quanxin, Distributed Coordinated Heterogeneous Task Allocation for Unmanned Aerial Vehicles, Control and Decision. 2 (2013) 274-278.

[2] Li Saihui, Lei Jinkui, Design and Implementation of Digital Rudder Control System Based on DSP, Computer Measurement and Control. 3 (2009) 484-486.

[3] Zhou Xiaoqing, Design and Implement of Electrical Actuator Control System of High Speed Unmanned Aerial Vehicle, Hangzhou: Zhejiang University, 2010.

[4] Kong Xiangji, Hou Wen, Chen Chunxi, Digital Rudder Control System Based on DSP, Electronic Test. 13 (2013) 31-32.

[5] Zhao Qiang, Liu Songfeng, Cheng Peng, Development and Key Technology in General Purpose Automatic Test System, Electronic Design and Engineering. 5 (2011) 160-162.

[6] Huang Shenglin, Shen Conghui, Sun Weichao, Core of NxTest: Synthetic Instrument System, Electronic Measurement Technology. 5 (2011) 64-67. 